\title{
Polymacromonomer Consisting of Polystyrene. Light Scattering Characterization in Cyclohexane
}

\author{
Ken Terao, Yoshishige Takeo, Masataka Tazaki, Yo Nakamura, \\ and Takashi NORISUYE
}

Department of Macromolecular Science, Osaka University,

Machikaneyama-cho 1-1, Toyonaka, Osaka 560-0043, Japan

(Received September 17, 1998)

\begin{abstract}
Nine samples of a polymacromonomer consisting of polystyrene with a fixed side-chain length of 33 styrene residues and ranging in total weight-average molecular weight $M_{w}$ from $1.9 \times 10^{5}$ to $1.1 \times 10^{7}$ have been prepared and studied by light scattering in cyclohexane at temperatures between 25 and $45^{\circ} \mathrm{C}$. The second virial coefficient is found to vanish at $34.5( \pm 2)^{\circ} \mathrm{C}$, the $\Theta$ temperature for linear polystyrene in cyclohexane. The $M_{w}$ dependence of $z$-average mean-square radius of gyration $\left\langle S^{2}\right\rangle_{z}$ at this temperature is analyzed on the basis of the wormlike chain to obtain $22 \mathrm{~nm}$ for Kuhn's statistical segment length $\lambda^{-1}$ and $13000 \mathrm{~nm}^{-1}$ for the molar mass per unit contour length. The chain stiffness as indicated by this $\lambda^{-1}$ value is one order of magnitude higher than that of linear polystyrene. Analysis of $\left\langle S^{2}\right\rangle_{z}$ at temperatures other than the $\Theta$ point is also made with the aid of the quasi-two-parameter theory for excluded-volume effects. It is found that $\lambda^{-1}$ increases gradually with raising temperature or increasing excluded-volume strength.

KEY WORDS Polymacromonomer / Branched Polymer / $\Theta$ Point / Wormlike Chain / Chain Stiffness / Excluded-Volume Effect /
\end{abstract}

Tsukahara et al. ${ }^{1-3}$ were the first to synthesize polymacromonomers consisting of the poly(methyl methacrylate) (PMMA) backbone and polystyrene (PS) side chains. These regular comb polymers have since called considerable attention of polymer physical chemists who are interested in molecular characterization of polymers in dilute solution. Analyses of dimensional and hydrodynamic data ${ }^{4-6}$ in toluene, a good solvent, based on the unperturbed wormlike chain ${ }^{7}$ show that the polymacromonomers studied are much stiffer than linear PMMA and that the Kuhn segment length $\lambda^{-1}$ becomes larger as the molecular weight of the PS side chain increases. This pronounced effect of branching on chain stiffness raises a new matter of interest to be explained in terms of interactions between the main chain and side chains and those between neighboring side chains in a polymer molecule. For fundamental studies of such intramolecular interactions use of polymacromonomers composed of single polymer species is desirable to avoid complexity arising from interactions between the PMMA and PS sub-chains.

In this work, we prepared nine samples of a polymacromonomer consisting only of PS and commenced their dilute-solution characterization by light scattering as a first step toward understanding the effects of molecular architecture and intramolecular interactions on chain stiffness. Figure 1 shows the chemical structure of the polymacromonomer, whose side chains have a fixed degree of polymerization $(n)$ of 33 . We note that Tsukahara et al. ${ }^{3,8}$ already synthesized similar polymacromonomers but reported no solution data.

At an early stage of the present study, we found that our polymacromonomer attains the $\Theta$ condition in cyclohexane around $34.5^{\circ} \mathrm{C}$, the $\Theta$ temperature for linear PS. This first example of the $\Theta$ state found for polymacromonomers prompted us to see by measurements of the $z$-average mean-square radius of gyration $\left\langle S^{2}\right\rangle_{z}$ how much the PS backbone is stiffened by the presence of long branches. In the work reported below, the $\left\langle S^{2}\right\rangle_{z}$ data in cyclohexane obtained as a function of molecular weight at temperatures between 32 and $45^{\circ} \mathrm{C}$ are analyzed on the basis of the wormlike chain ${ }^{7,9}$ to estimate $\lambda^{-1}$.

\section{EXPERIMENTAL}

Samples

$\alpha$-Benzyl- $\omega$-vinylbenzyl polystyrene, a macromonomer, was synthesized by living anionic polymerization in the manner reported by Tsukahara et al. ${ }^{3,8}$ The product was reprecipitated 10 times from benzene solutions into methanol to remove unreacted $p$-chloromethylstyrene. Its molecular weight was $3.56 \times 10^{3}$ when estimated from the weight-average molecular weight $M_{w}$ of $3.44 \times 10^{3}$ determined for the precursor ( $\alpha$-benzyl- $\omega$-hydrogen polystyrene) by light scattering. The weight-average to number-average molecular weight ratio $M_{w} / M_{n}$ for the precursor was estimated to be 1.03 by a MALDITOF spectrometer with a trans, trans-1,4-diphenyl-1,3butadiene matrix containing silver ions in form of $\mathrm{AgCF}_{3} \mathrm{COO}$; the value of $M_{w}$ evaluated from the mass spectrum agreed with the light scattering value of $3.44 \times 10^{3}$ within $3 \%$. The macromonomer was polymerized in benzene at $45-60^{\circ} \mathrm{C}$ for $50-100 \mathrm{~h}$ with azobis(isobutyronitrile) as an initiator to obtain 11 polymacromonomer samples.

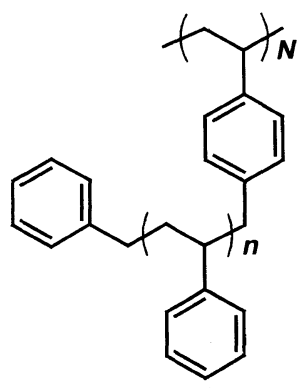

Figure 1. Chemical structure of a polymacromonomer consisting of polystyrene. The main chain and each side chain have degrees of polymerization of $N$ and $n$, respectively. 
After unreacted macromonomers had been removed by fractional solution, the products were divided each into a number of parts by repeated fractional precipitation with toluene as the solvent and methanol as the precipitant, or with cyclohexane at successively varying temperatures; when cooled below $\Theta$, cyclohexane solutions separated into two phases, and the phase separation was effective for fractionation of high molecular weight samples. From the fractions obtained, 9 middle ones designated below as F33-1, F33-2, F33-3, F33-4, F33-6, F33-7, F33-9, F33-11, and F33-12 were chosen for the present study. These fractions were freeze-dried from benzene solutions. Values of $M_{w} / M_{n}$ for some of them were estimated by gel permeation chromatography (GPC).

\section{Light Scattering}

Light scattering measurements were made on a Fica-50 light scattering photometer with vertically polarized incident light of 436 and 546-nm wavelengths in an angular range from 22.5 to $150^{\circ}$. The apparatus was calibrated with benzene at $25^{\circ} \mathrm{C}$ as the reference liquid, whose Rayleigh ratios at 436 and $546 \mathrm{~nm}$ were taken to be $46.5 \times 10^{-6}$ and $16.1 \times 10^{-6} \mathrm{~cm}^{-1}$, respectively. ${ }^{10}$ The depolarization ratio of this liquid was determined to be 0.41 and 0.40 for 436 and $546 \mathrm{~nm}$, respectively, by the method of Rubingh and Yu. ${ }^{11}$

The optical anisotropy of our polymacromonomer was investigated for samples F33-4, F33-7, and F33-12 in cyclohexane at different temperatures. When the light scattering envelopes observed with the analyzer set in the vertical direction and without analyzer were compared, no difference was detected for any of these samples. Furthermore, the scattered intensity of the lowest molecular weight sample F33-12 measured with the analyzer set in the horizontal direction was not more than $0.4 \%$ of the intensity measured with no analyzer, at every angle studied. From these results the optical anisotropy of the polymacromonomer molecule was concluded to be negligible in the range of molecular weight studied, and no anisotropy correction was applied to any of light scattering data obtained in this work.

Polymer solutions and the solvent (cyclohexane) were made optically clean by centrifugation at about $3 \times 10^{4}$ gravities for $1.5 \mathrm{~h}$. Each of them was transferred directly into the light scattering cell with a carefully cleaned pipet.

The specific refractive index increment $\partial n / \partial c$ of the polymacromonomer in cyclohexane was determined at $25,30,35$, and $40^{\circ} \mathrm{C}$ using a modified Schulz-Cantow type differential refractmeter. It was found to be represented by

$$
\begin{array}{r}
\partial n / \partial c=4.0 \times 10^{-4} T\left({ }^{\circ} \mathrm{C}\right)+0.168 \\
\left(\mathrm{~cm}^{3} \mathrm{~g}^{-1} ; \lambda_{0}=436 \mathrm{~nm}\right) \\
\partial n / \partial c=4.0 \times 10^{-4} T\left({ }^{\circ} \mathrm{C}\right)+0.156 \\
\left(\mathrm{~cm}^{3} \mathrm{~g}^{-1} ; \lambda_{0}=546 \mathrm{~nm}\right)
\end{array}
$$

at polymer mass concentrations $c$ lower than $1 \times 10^{-2}$ $\mathrm{g} \mathrm{cm}^{-3}$.

\section{RESULTS}

Figure 2 illustrates the concentration dependence of

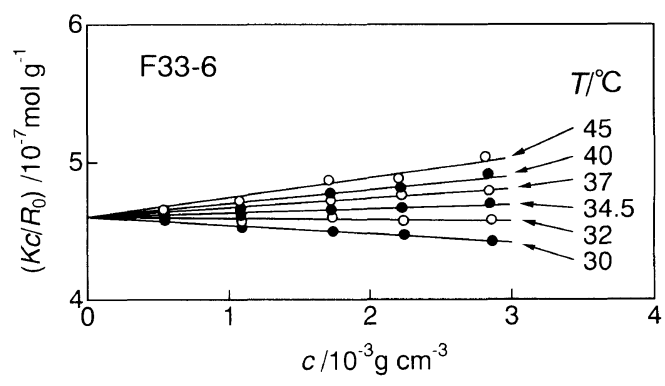

Figure 2. Concentration dependence of $K c / R_{0}$ for polymacromonomer sample F33-6 in cyclohexane at indicated temperatures.

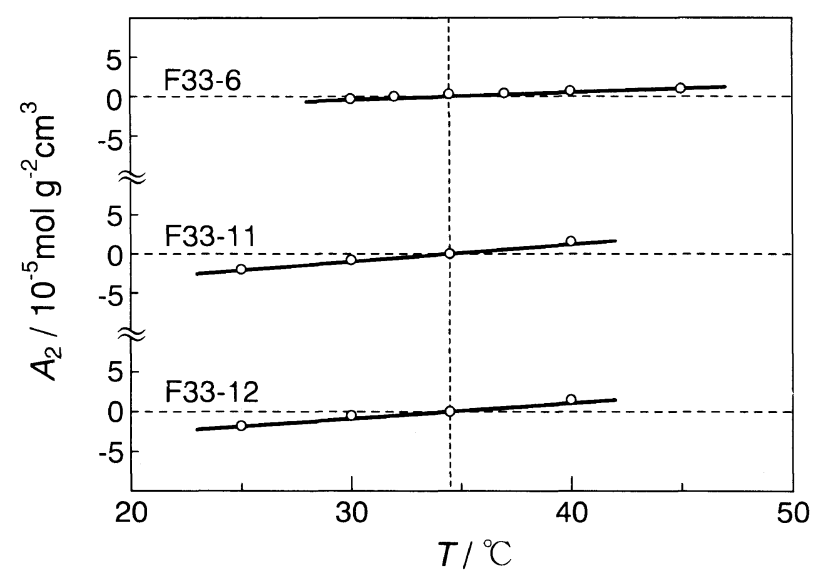

Figure 3. Temperature dependence of $A_{2}$ for polymacromonomer samples F33-6, F33-11, and F33-12 in cyclohexane. The vertical dashed line indicates $34.5^{\circ} \mathrm{C}$ (the $\Theta$ temperature).

$K c / R_{0}$ for sample F33-6 in cyclohexane at the indicated temperatures $T$, where $K$ and $R_{0}$ denote the optical constant and the excess reduced scattering intensity at zero angle, respectively. The $K c / R_{0}$ values have been obtained by extrapolation of plots of $\left(K c / R_{\theta}\right)^{1 / 2} v s$. $\sin ^{2}(\theta / 2)$ to $\theta=0\left(R_{\theta}\right.$ is the excess reduced scattering intensity at scattering angle $\theta$ ). The straight lines fitting the plotted points at the respective $T$ converge to a common intercept, yielding an identical $M_{w}$ within experimental error. The slope and hence the second virial coefficient $A_{2}$ change from negative to positive with increasing $T$. This can be seen more clearly in Figure 3, in which the $A_{2}$ data for the sample are plotted against $T$, along with those for samples F33-11 and F33-12. The virial coefficients for the three samples vanish around $34.5^{\circ} \mathrm{C}$, the $\Theta$ temperature for linear PS in cyclohexane. Thus we find that our polymacromonomer in cyclohexane happens to have a $\Theta$ point at essentially the same temperature as that for the linear chain. With regard to the precision of the $\Theta$ temperature for the present system, the following remark may be pertinent.

As can be seen in Figure 3, the $T$-dependence of $A_{2}$ is very weak (compared to that for linear $\mathrm{PS}^{12}$ ). In particular, $A_{2}$ for sample F33-6 stays on the order of $10^{-6} \mathrm{~mol} \mathrm{~g}^{-2} \mathrm{~cm}^{3}$ over a range of $T$ from 32 to $37^{\circ} \mathrm{C}$. Such a $T$ range extended more for a higher $M_{w}$ (not shown here). In view of the $T$-insensitive behavior of $A_{2}$, it is probably safe to allow of an uncertainty of about $\pm 2^{\circ} \mathrm{C}$ for the $\Theta$ point $\left(34.5^{\circ} \mathrm{C}\right)$ of the polymacromonomer + cyclohexane system.

Figure 4 shows the plots of $P(\theta)^{-1 / 2}$ vs. $\sin ^{2}(\theta / 2)$ for all the samples except F33-11 and F33-12 in cyclohexane 


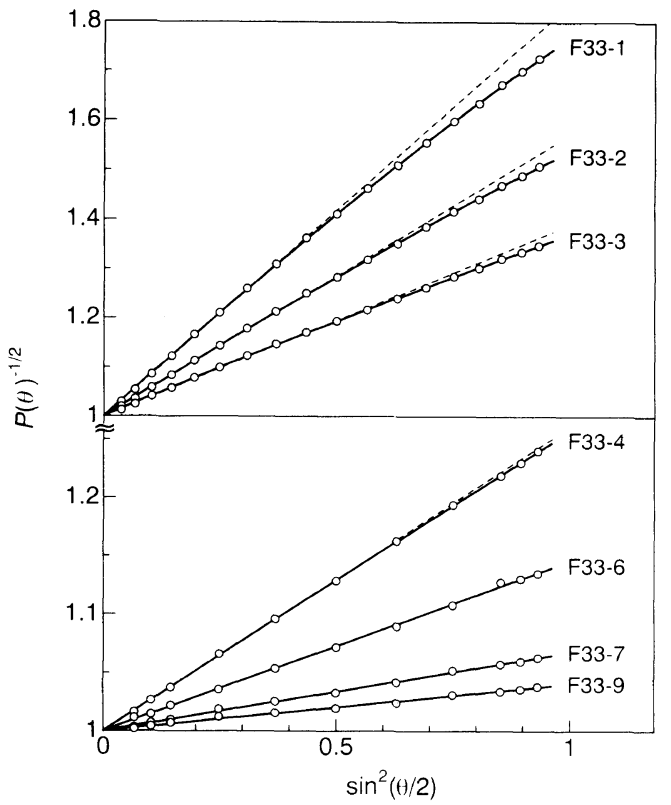

Figure 4. Angular dependence of $P(\theta)^{-1 / 2}$ for indicated polymacromonomer samples in cyclohexane at $34.5^{\circ} \mathrm{C}$. Dashed lines, initial slopes; wavelength of incident light, $436 \mathrm{~nm}$.

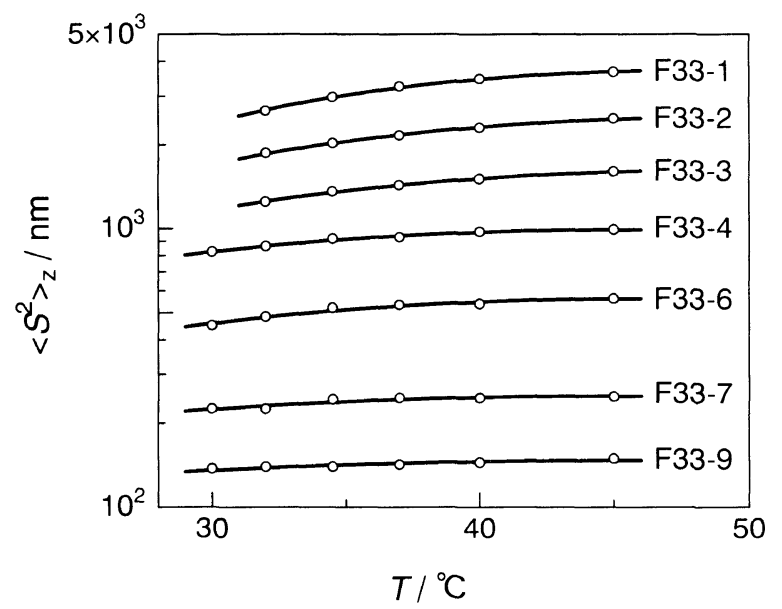

Figure 5. Temperature dependence of $\left\langle S^{2}\right\rangle_{z}$ for the indicated samples in cyclohexane.

at $34.5^{\circ} \mathrm{C}$, where $P(\theta)$ denotes the particle scattering function. The values of $\left\langle S^{2}\right\rangle_{z}$ evaluated from the initial slopes (the dashed lines) are summarized in Table I, along with those at other temperatures and data for $M_{w}$ and $M_{w} / M_{n}$. The $M_{w} / M_{n}$ values indicate that our samples examined by GPC are all narrow in molecular weight distribution.

The temperature dependence of $\left\langle S^{2}\right\rangle_{z}$ for seven samples is illustrated in Figure 5. It is much weaker than that ${ }^{13}$ for linear PS with the same molecular weight in cyclohexane.

Figure 6 shows the molecular weight dependence of $\left\langle S^{2}\right\rangle_{z}$ for our polymacromonomer in cyclohexane at $\Theta$ $\left(34.5^{\circ} \mathrm{C}\right)$. The curve bends down and has a slope 1.4 for $M_{w}<2 \times 10^{6}$ and 1.05 for $M_{w}>5 \times 10^{6}$. This nonGaussian behavior shows an unmistakable semiflexibility of the polymer.

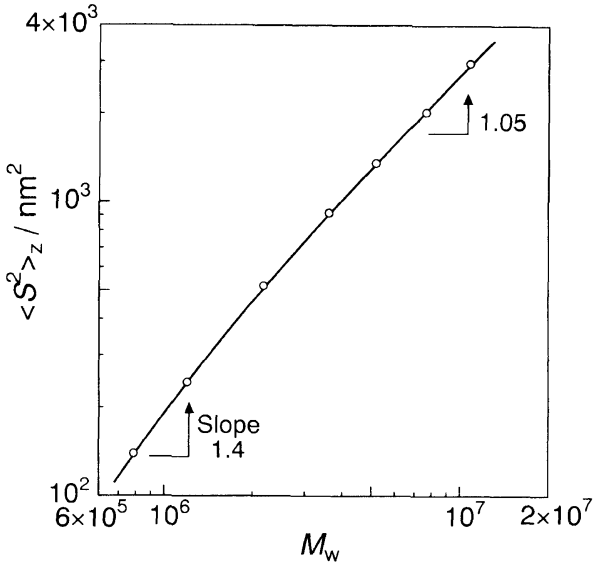

Figure 6. Molecular weight dependence of $\left\langle S^{2}\right\rangle_{2}$ for the polymacromonomer in cyclohexane at $34.5^{\circ} \mathrm{C}$. The curve represents eq 1 with $\lambda^{-1}=22 \mathrm{~nm}$ and $M_{\mathrm{L}}=13000 \mathrm{~nm}^{-1}$.

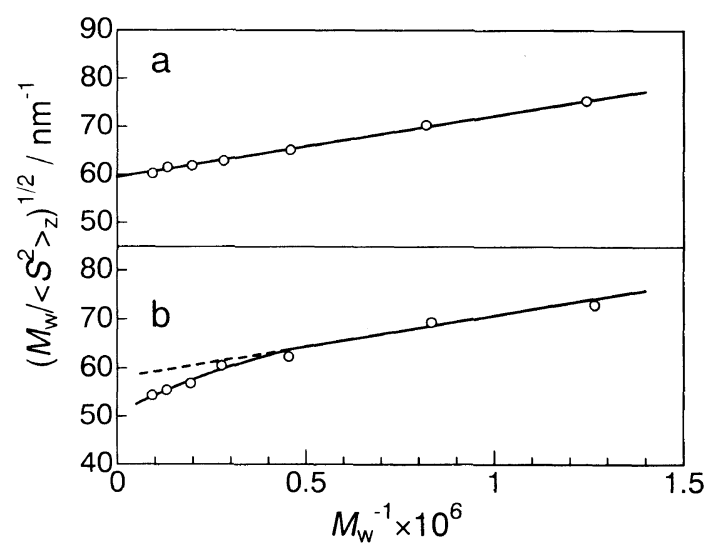

Figure 7. Plots of $\left(M_{w} /\left\langle S^{2}\right\rangle_{z}\right)^{1 / 2} v s . M^{-1}$ for the polymacromonomer in cyclohexane at the $\Theta$ temperature (a) and at $45^{\circ} \mathrm{C}$ (b)

\section{DISCUSSION}

\section{Data Analysis}

(i) At the $\Theta$ Temperature. If our polymacromonomer in cyclohexane is modeled by the wormlike chain, ${ }^{7}$ its $\left\langle S^{2}\right\rangle$ in the unperturbed state, i.e., $\left\langle S^{2}\right\rangle_{0}$, may be expressed by ${ }^{14}$

$$
\lambda^{2}\left\langle S^{2}\right\rangle_{0}=\frac{\lambda L}{6}-\frac{1}{4}+\frac{1}{4 \lambda L}-\frac{1}{8(\lambda L)^{2}}[1-\exp (-2 \lambda L)]
$$

Here, $L$ is the contour length of the main chain related to the molecular weight $M$ by $L=M / M_{\mathrm{L}}$, with $M_{\mathrm{L}}$ being the molar mass per unit contour length. According to Murakami et al., ${ }^{15}$ eq 1 can be approximated by

$$
\left(\frac{M}{\left\langle S^{2}\right\rangle_{0}}\right)^{1 / 2}=\left(6 \lambda M_{\mathrm{L}}\right)^{1 / 2}\left(1+\frac{3 M_{\mathrm{L}}}{4 \lambda M}\right)
$$

with the maximum error from the exact value being $1 \%$ for $\lambda L>2$. Equation 2 indicates that $\left(M /\left\langle S^{2}\right\rangle_{0}\right)^{1 / 2}$ plotted against $M^{-1}$ should give a straight line whose intercept and slope allow the two parameters $\lambda^{-1}$ and $M_{\mathrm{L}}$ to be evaluated.

Figure 7a shows this plot constructed from our $\left\langle S^{2}\right\rangle_{z}$ data in cyclohexane at the $\Theta$ temperature. The data points follow a straight line, which yields $(22.0 \pm 0.6) \mathrm{nm}$ for 
$\lambda^{-1}$ and $(13000 \pm 300) \mathrm{nm}^{-1}$ for $M_{\mathrm{L}}$. The curve in Figure 6 represents the theoretical values calculated from eq 1 with $\lambda^{-1}=22 \mathrm{~nm}$ and $M_{\mathrm{L}}=13000 \mathrm{~nm}^{-1}$. Its fit to the data points is excellent. The $\lambda^{-1}$ value of $22 \mathrm{~nm}$ shows a considerable stiffness of the polymer, but it is not as large as that $(89 \mathrm{~nm})$ reported for the polymacromonomer consisting of the PMMA backbone and 28 styrene side-chain residues in toluene. ${ }^{4-6}$

In the above analysis, we ignored possible effects of chain thickness and chain ends on $\left\langle S^{2}\right\rangle_{0}$; note that the latter effect may arise if side chains near the ends of the main chain are more or less oriented to the direction of the main-chain contour to apparently increase the contour length. We examined these effects by evaluating the mean-square radius of gyration $\left\langle S^{2}\right\rangle_{0, t}$ of a wormlike polymacromonomer whose side chains are flexible (but yet wormlike) and linked to the stiffer backbone by completely flexible joints. The resulting expression is the sum of $\left\langle S^{2}\right\rangle_{0}$ of the backbone (i.e., eq 1) and the contribution $\left\langle S^{2}\right\rangle_{0, \mathrm{~s}}$ from the side chains, and the latter is a function of the number of side chains (equal to the degree of polymerization of the main chain), $\lambda_{\mathrm{s}}^{-1}$ (Kuhn's segment length of each side chain), and $L_{\mathrm{s}}$ (the side-chain contour length equal to $M_{\mathrm{s}} / M_{\mathrm{L}, \mathrm{s}}$, with $M_{\mathrm{s}}$ and $M_{\mathrm{L}, \mathrm{s}}$ being the molar mass of the precursor and the molar mass per unit contour length of each side chain, respectively); the expression is not shown here since it is a little lengthy and its derivation is elementary.

The $\left\langle S^{2}\right\rangle_{0, s}$ computed as a function of the molecular weight $M$ of the polymacromonomer with $M_{\mathrm{s}}=3.44 \times$ $10^{3}, \lambda_{\mathrm{s}}^{-1}=2.0 \mathrm{~nm}$, and $M_{\mathrm{L}, \mathrm{s}}=390 \mathrm{~nm}^{-1}$ (the parameters for linear $\mathrm{PS}^{16}$ ) was about $7 \mathrm{~nm}^{2}$, almost regardless of $M$ above $2 \times 10^{5}$, and its contribution to $\left\langle S^{2}\right\rangle_{0, t}$ was $2.9 \%$ for $M=1.22 \times 10^{6}$ and $5 \%$ even for $M=8.0 \times 10^{5}$, the lowest molecular weight with which we are concerned here. Since those contributions are hardly beyond experimental errors of $\left\langle S^{2}\right\rangle_{z}$, we convince ourselves that the present $\left\langle S^{2}\right\rangle_{z}$ data are essentially free from the above-mentioned effects of side chains and that the above analysis based on eq 1 introduces no significant error in the wormlike-chain parameters.

(ii) Near the $\Theta$ Temperature. Our $\left\langle S^{2}\right\rangle_{z}$ data near $\Theta$ do not always obey the linear relation of $\left(M_{w} /\left\langle S^{2}\right\rangle_{z}\right)^{1 / 2}$ vs. $M_{w}{ }^{-1}$. An example is shown with the data at $45^{\circ} \mathrm{C}$ in Figure $7 \mathrm{~b}$. The pronounced downward deviations of plotted points from the dashed line may be ascribed to excluded-volume effects on $\left\langle S^{2}\right\rangle_{z}$ at this temperature. In the following, we analyze $\left\langle S^{2}\right\rangle_{z}$ data near $\Theta$ with the aid of the quasi-two-parameter (QTP) theory ${ }^{9}$ for wormlike $^{7}$ or helical wormlike ${ }^{9}$ bead chains.

In the QTP scheme, ${ }^{9,17,18}$ the radius expansion factor $\alpha_{\mathrm{s}}\left[\equiv\left(\left\langle S^{2}\right\rangle /\left\langle S^{2}\right\rangle_{0}\right)^{1 / 2}\right]$ is a universal function of the scaled excluded-volume parameter $\tilde{z}$ defined by

$$
\tilde{z}=\frac{3}{4} K(\lambda L) z
$$

with

$$
z=\left(\frac{3}{2 \pi}\right)^{3 / 2}(\lambda B)(\lambda L)^{1 / 2}
$$

and

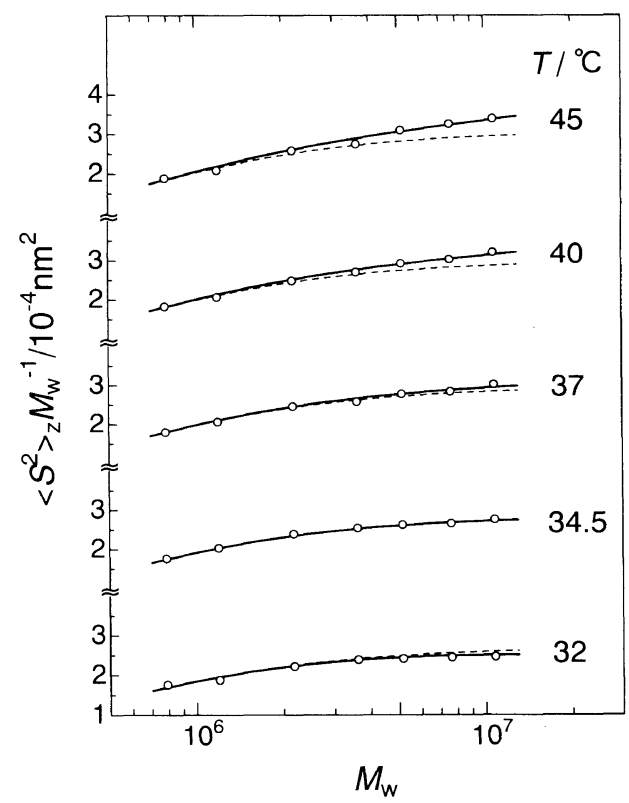

Figure 8. Comparison between the measured $\left\langle S^{2}\right\rangle_{z} M_{w}{ }^{-1}$ for the polymacromonomer in cyclohexane and the theoretical values (solid line) calculated from eq 1 and 7 with the parameters given in Table II. Dashed lines, theoretical values for $B=0$.

$$
\begin{aligned}
& K(\lambda L) \\
& =\frac{4}{3}-2.711(\lambda L)^{-1 / 2}+\frac{7}{6}(\lambda L)^{-1} \text { for } \lambda L>6 \\
& =(\lambda L)^{-1 / 2} \exp \left[-6.611(\lambda L)^{-1}+0.9198+0.03516 \lambda L\right] \\
& \text { for } \lambda L<6
\end{aligned}
$$

Here, $z$ is the conventional excluded-volume parameter and $B$ is the excluded-volume strength defined for the wormlike chain by

$$
B=\beta / a^{2}
$$

with $\beta$ and $a$ being the binary cluster integral representing the interaction between a pair of beads and the bead spacing, respectively. Note that one bead must contain a number of main-chain residues in our case.

Adopting the Domb-Barrett function ${ }^{19}$ for $\alpha_{\mathrm{s}}^{2}$, we have

$$
\begin{aligned}
\alpha_{\mathrm{s}}^{2}= & {\left[1+10 \tilde{z}+\left(\frac{70 \pi}{9}+\frac{10}{3}\right) \tilde{z}^{2}+8 \pi^{3 / 2} \tilde{z}^{2}\right]^{2 / 15} } \\
& \times\left[0.933+0.067 \exp \left(-0.85 \tilde{z}-1.39 \tilde{z}^{2}\right)\right]
\end{aligned}
$$

This equation is known to accurately describe experimental data for linear flexible and semiflexible polymers over a broad range of molecular weight. ${ }^{20-24}$ Equations 3 through 7 indicate that $B$ needs to be known (in addition to $\lambda^{-1}$ and $M_{\mathrm{L}}$ characterizing the unperturbed wormlike chain) in order to evaluate the theoretical $\alpha_{\mathrm{s}}^{2}$ and hence the theoretical $\left\langle S^{2}\right\rangle$ in the perturbed state. Although eq 7 has been constructed for $\tilde{z}>0$, it should be applicable to very small negative $\tilde{z}$ too.

A curve fitting procedure was employed for our analysis, but $M_{\mathrm{L}}$ at any $T$ was assumed to be the same as that at $34.5^{\circ} \mathrm{C}$; we note that the three parameters could not uniquely be determined from the $\left\langle\mathrm{S}^{2}\right\rangle_{z}$ data because of the correlation between $\lambda^{-1}$ and $B$ in the range of molecular weight studied. The parameters ob- 
Polymacromonomer Consisting of Polystyrene

Table I. Results from light scattering and GPC measurements on polymacromonomer samples in cyclohexane at different temperatures

\begin{tabular}{|c|c|c|c|c|c|c|c|c|}
\hline \multirow{2}{*}{ Sample } & \multirow{2}{*}{$M_{w} / 10^{5}$} & \multicolumn{6}{|c|}{$\left\langle S^{2}\right\rangle_{z} / 10^{2} \mathrm{~nm}^{2}$} & \multirow{2}{*}{$M_{w} / M_{n}$} \\
\hline & & $30^{\circ} \mathrm{C}$ & $32^{\circ} \mathrm{C}$ & $34.5^{\circ} \mathrm{C}$ & $37^{\circ} \mathrm{C}$ & $40^{\circ} \mathrm{C}$ & $45^{\circ} \mathrm{C}$ & \\
\hline F33-1 & 106 & & 26.5 & 29.7 & 32.5 & 34.6 & 36.7 & \\
\hline F33-2 & 75.0 & & 18.6 & 20.2 & 21.6 & 23.0 & 24.9 & 1.10 \\
\hline F33-3 & 50.5 & & 12.5 & 13.6 & 14.3 & 15.0 & 16.0 & 1.08 \\
\hline F33-4 & 35.5 & 8.21 & 8.63 & 9.21 & 9.33 & 9.71 & 9.90 & 1.06 \\
\hline F33-6 & 21.8 & 4.51 & 4.83 & 5.21 & 5.34 & 5.38 & 5.62 & 1.06 \\
\hline F33-7 & 12.2 & 2.25 & 2.26 & 2.43 & 2.46 & 2.46 & 2.50 & 1.04 \\
\hline F33-9 & 8.03 & 1.37 & 1.39 & 1.39 & 1.42 & 1.44 & 1.49 & 1.05 \\
\hline F33-11 & 3.00 & & & & & & & \\
\hline F33-12 & 1.89 & & & & & & & \\
\hline
\end{tabular}

tained are summarized in Table II. Figure 8 shows that the theoretical solid curves calculated with these parameters closely fit the data points at the respective temperatures throughout the entire range of molecular weight examined. The dashed line for each $T$ refers to the unperturbed state (i.e., $B=0$ ). At any temperatures except $34.5^{\circ} \mathrm{C}$, excluded-volume effects are appreciable for $M_{w}$ above $3 \times 10^{6}$ but negligible below that $M_{w}$. If we rely on the $\left\langle S^{2}\right\rangle_{z}$ data for $M_{w}<3 \times 10^{6}$ and analyze them (at the respective $T$ ) using eq 2 for the unperturbed wormlike chain ( $c f$. the dashed line in Figure 7b), we obtain essentially the same $M_{\mathrm{L}}$ values as $13000 \mathrm{~nm}^{-1}$ (the value at $34.5^{\circ} \mathrm{C}$ ) and $\lambda^{-1}$ values indistinguishable from those given in Table II. This finding lends support to the above assumption that $M_{\mathrm{L}}$ of the polymacromonomer in cyclohexane near $\Theta$ is essentially independent of $T$.

\section{Molecular Characteristics}

The estimated $M_{\mathrm{L}}$ value of $13000 \mathrm{~nm}^{-1}$ (at the $\Theta$ temperature) with the molar mass (3560) of the macromonomer yields $0.27 \mathrm{~nm}$ for the contour length per main-chain residue. This monomeric length is close to that $(0.25 \mathrm{~nm})$ calculated on the assumption that the polystyrene backbone assumes the all-trans conformation. The small difference between these values should diminish if the $\left\langle S^{2}\right\rangle_{z}$ data are corrected for polydispersity (see Table I). In the present work, however, no such elaborate analysis was made.

The $\lambda^{-1}$ value of $22 \mathrm{~nm}$ at $34.5^{\circ} \mathrm{C}$ is one order of magnitude larger than that for the linear PS molecule in cyclohexane at the same temperature $\left(\lambda^{-1}=2 \mathrm{~nm}\right.$ if modeled by the wormlike chain) ${ }^{16}$ note that linear PS is better modeled by the helical wormlike chain ${ }^{9}$ and that $\lambda^{-1}$ (the stiffness parameter) in this model is $2.1-$ $2.7 \mathrm{~nm} .^{9,25-28}$ The much larger $\lambda^{-1}$ for the polymacromonomer implies that the high segment density around the main chain significantly restricts the conformational freedom of backbone residues, thereby stiffening the polymer chain. This idea was early given by Noda et al., ${ }^{29}$ who found that comb-shaped polystyrenes with different numbers of branching in cyclohexane are stiffer (in the modern terminology) than linear PS. Interestingly, this group observed that the $\Theta$ temperatures of those comb-shaped polymers are not very different from that for the linear chain. This resembles what we have found here, though Noda et al.'s samples have much lower degrees of branching. It should be noted that the precise $\Theta$ temperature may vary
Table II. Wormlike-chain parameters and excluded-volume strength for the polymacromonomer in cyclohexane at different temperatures

\begin{tabular}{lccc}
\hline$T /{ }^{\circ} \mathrm{C}$ & $\lambda^{-1} / \mathrm{nm}$ & $M_{\mathrm{L}} / 10^{4} \mathrm{~nm}^{-1}$ & $B / \mathrm{nm}$ \\
\hline 32 & 21 & $1.30^{\mathrm{a}}$ & -0.4 \\
34.5 & 22 & 1.30 & 0 \\
37 & 23 & $1.30^{\mathrm{a}}$ & 0.5 \\
40 & 23 & $1.30^{\mathrm{a}}$ & 1.5 \\
45 & 24 & $1.30^{\mathrm{a}}$ & 2.4 \\
\hline
\end{tabular}

${ }^{\text {a Assumed. }}$

depending on the kind of chemical species of the end groups in each side chain.

As can be seen from Table II, both $\lambda^{-1}$ and $B$ increase gradually with raising temperature. This finding suggests that enhancement of side chain-side chain or main chain-side chain repulsion causes the polymer backbone to be stiffened; note that by its definition (i.e., the bending force constant divided by the product of the Boltzmann constant and $T$ ), $\lambda^{-1}$ may generally be expected to decrease with raising $T$. It is thus intriguing to see whether our polymacromonomer has a higher stiffness in such a good solvent as toluene than in the $\Theta$ solvent. Work on this problem is in progress in this laboratory and will be reported shortly, along with that done for another polymacromonomer with 15 styrene side-chain residues.

\section{CONCLUSIONS}

A polymacromonomer consisting of polystyrene with 33 side-chain residues attains the $\Theta$ state in cyclohexane at $34.5^{\circ} \mathrm{C}$, as is the case with linear polystyrene. Analysis of $\left\langle S^{2}\right\rangle_{z}$ data in cyclohexane at this temperature shows the polymacromonomer to be characterized by the wormlike chain with a Kuhn segment length of $22 \mathrm{~nm}$ and a linear mass density of $13000 \mathrm{~nm}^{-1}$. This Kuhn length (or more generally the stiffness parameter in the helical wormlike chain) is one order of magnitude higher than that for the linear chain, indicating that the polystyrene backbone is remarkably stiffened by the presence of the long branches. At temperatures (32$45^{\circ} \mathrm{C}$ ) other than $\Theta$, excluded-volume effects on $\left\langle S^{2}\right\rangle_{z}$ are not negligible unless $M_{w}$ is lower than $3 \times 10^{6}$, and the chain stiffness tends to increase with raising temperature, probably owing to the enhancement of repulsion between neighboring side chains as well as that 
between the main chain and side chains.

Acknowledgment. We are very grateful to Professor Jimmy W. Mays of the University of Alabama at Birmingham for his help in MALDI-TOF-mass spectroscopy. Y. N. thanks Dr. Atsushi Takano of Nagaoka University of Technology for helpful discussions on the synthesis of polystyrene macromonomers.

\section{REFERENCES}

1. Y. Tsukahara, K. Mizuno, A. Segawa, and Y. Yamashita, Macromolecules, 22, 1546 (1989).

2. Y. Tsukahara, K. Tsutsumi, Y. Yamashita, and S. Shimada, Macromolecules, 23, 5201 (1990).

3. Y. Tsukahara, in "Macromolecular Design: Concept and Practice," M. K. Mishra, Ed., Polymer Frontiers International Inc., New York, N.Y., 1994, pp 161-227.

4. M. Wintermantel, M. Schmidt, Y. Tsukahara, K. Kajiwara, and S. Kohjiya, Macromol. Rapid Commun., 15, 279 (1994).

5. M. Wintermantel, M. Gerle, K. Fischer, M. Schmidt, I. Wataoka, H. Urakawa, K. Kajiwara, and Y. Tsukahara, Macromolecules, 29, 978 (1996).

6. N. Nemoto, M. Nagai, A. Koike, and S. Okada, Macromolecules, 28, 3854 (1995).

7. O. Kratky and G. Porod, Recl. Trav. Chim. Pays-bas, 68, 1106 (1949).

8. Y. Tsukahara, J. Inoue, Y. Ohta, S. Kohjiya, and Y. Okamoto, Polym. J., 26, 1013 (1994).

9. H. Yamakawa, "Helical Wormlike Chains in Polymer Solutions," Springer, Berlin, 1997.

10. Gj. Dezželić and J. Vavra, Croat. Chem. Acta, 38, 35 (1966).
11. D. N. Rubingh and H. Yu, Macromolecules, 9, 681 (1976).

12. See, for example, Y. Nakamura, T. Norisuye, and A. Teramoto, Macromolecules, 24, 4904 (1991).

13. See, for example, Y. Miyaki, Ph.D. Thesis, Osaka University, 1981.

14. H. Benoit and P. Doty, J. Phys. Chem., 57, 958 (1953).

15. H. Murakami, T. Norisuye, and H. Fujita, Macromolecules, 13, 345 (1980).

16. T. Norisuye and H. Fujita, Polym. J., 14, 143 (1982).

17. H. Yamakawa and W. H. Stockmayer, J. Chem. Phys., 57, 2843 (1972).

18. J. Shimada and H. Yamakawa, J. Chem. Phys., 85, 591 (1986).

19. C. Domb and A. J. Barrett, Polymer, 17, 179 (1976).

20. F. Abe, Y. Einaga, T. Yoshizaki, and H. Yamakawa, Macromolecules, 26, 1884 (1993).

21. K. Horita, F. Abe, Y. Einaga, and H. Yamakawa, Macromolecules, 26, 5067 (1993).

22. F. Abe, K. Horita, Y. Einaga, and H. Yamakawa, Macromolecules, 27, 725 (1994)

23. M. Kamijo, F. Abe, Y. Einaga, and H. Yamakawa, Macromolecules, 28, 1095 (1995).

24. T. Norisuye, A. Tsuboi, and A. Teramoto, Polym. J., 28, 357 (1996).

25. Y. Einaga, H. Koyana, T. Konishi, and H. Yamakawa, Macromolecules, 22, 3419 (1989).

26. T. Konishi, T. Yoshizaki, T. Saito, Y. Einaga, and H. Yamakawa, Macromolecules, 23, 290 (1990).

27. T. Konishi, T. Yoshizaki, and H. Yamakawa, Macromolecules, 24, 5614 (1991).

28. T. Yamada, T. Yoshizaki, and H. Yamakawa, Macromolecules, 25, 377 (1992)

29. I. Noda, T. Horikawa, T. Kato, T. Fujimoto, and M. Nagasawa, Macromolecules, 3, 795 (1970). 